\title{
Public Health Messaging in Verón, Dominican Republic
}

\author{
Rebekah Schwartz ${ }^{1}$ and Jocelyn Widmer ${ }^{1,2}$ \\ ${ }^{l}$ College of Public Health \& Health Professions, University of Florida \\ ${ }^{1,2}$ College of Design, Construction and Planning ${ }^{2}$, University of Florida
}

Faculty mentor: Jocelyn Widmer, Department of Urban and Regional Planning

\begin{abstract}
The purpose of this project was to create public health programming that focused on prevention techniques of the most common health issues that present among Verón's child population. In May 2017, Fundación Grupo Puntacana opened a new children's clinic in the informal urban area of Verón. Through the Oscar de la Renta Pediatric Clinic in Verón, we created public health messages to play in the waiting room. We collaborated with the children's clinic doctors, had informal conversations with community members and leaders, and observed the operations of the children's clinic to gain a better understanding of children's health issues. Through visiting various neighborhoods of Verón, we filmed children and parents for our prevention video to demonstrate preventive techniques in the community context. Our goal was to create videos that encompass cultural sensitivities of the region as well as convey a clear health education message to parents and guardians. After the videos were created, we presented the videos to the doctors, various community members, and Fundación Grupo Puntacana representatives. When creating public health messaging for targeted audiences in a community that boasts a great deal of diversity, cultural integration is important to consider in creating effective content. In addition, it is important to have conversations with various parties to obtain a full understanding of social, economic, and other factors that affect the health of the community's children.
\end{abstract}

\section{Introduction}

Through the evolution and globalization of technology, public health messaging has grown and become an avenue to disseminate information and facilitate health education to wide audiences. In 2014, the West Africa Ebola Outbreak spread at alarming rates, affecting thousands of people in multiple communities. With increasing mortality rates, health departments and health officials needed to send important health education announcements and information to the people. For efficient delivery of messages, local health departments began to utilize channels such as social media platforms, like Twitter, to disseminate information and provide warnings to a greater audience (Wong, Harris, Staub, \& Bernhardt, 2017). In 2017, short messaging systems, or SMS, were created to expand health education to improve reproductive health outcomes in Kenya. These films were a method for women to discuss sexual health topics with their partners (Lewis, 2017). As seen in these two examples, public health messaging is becoming a tool around the 
globe to empower individuals to disseminate information and health education in a timely and comprehensive manner. Additionally, public health messaging is shown to be positively correlated with assisting with positive behavior change (De Leon, Fuentes, \& Cohen, 2014). One form of public health messaging that has grown has been short films. To address health concerns within the community, public health practitioners can utilize public health messaging in the form of films to target communities and improve health outcomes.

Moreover, when creating public health messaging it is important to recognize factors that affect people on an individual, relationship, community and societal level (Golden \& Earp, 2012), or also known as the Social Ecological Model. When creating content for messaging, it is crucial to recognize that individuals are influenced by factors such as socioeconomic status, government, physical environment, family, culture, and personal beliefs. These factors play a role in one's decisions and behaviors, particularly related to their health. With public health messaging, it is imperative that factors are explored an all levels from personal to societal to ensure the messaging will have the greatest impact and create effective behavior change (Golden \& Earp, 2012).

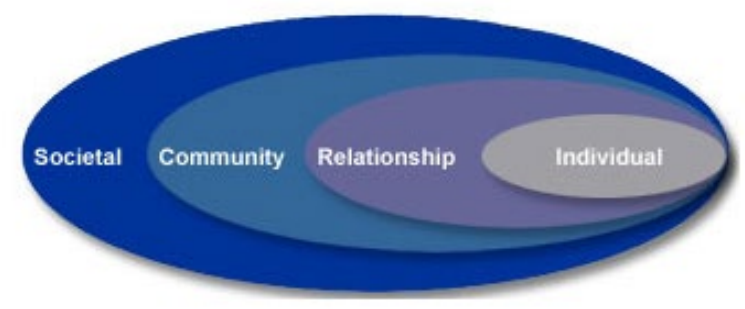

Figure 1: Social Ecological Model. Reprinted from Center for Disease Control and Prevention, 2015.

\section{Background}

The Dominican Republic is one of the two nations that compose the island of Hispaniola in the Caribbean Sea. The country has a population of 10.5 million people with a tenth of the population under the age of 5 (Human Development Reports, 2016). The infant mortality rate of the country is 25.7 per 1,000 live births (Human Development Reports, 2016) with only $2.9 \%$ of the GDP allotted to public health expenditure (Human Development Reports, 2016). For our research site, we specifically worked in Verón, Dominican Republic, located on the Northeastern portion of the island. 
Verón is an informal urban settlement in the Dominican Republic that is located adjacent to tourist development in the Punta Cana region. Verón is split into 24 sectors, which serve as communities, or neighborhoods, that compose the urban area. While there is little literature regarding this community, for the past three years, a group from the University of Florida has conducted various research studies within Verón. In 2016, a survey was administered to collect data at the community level. The survey provided context about the major health concerns, health education, source of drinking water, employment, migration flows, housing, and governance. According to the survey, health concerns included accidents and diseases such as dermatological, fever, gastrointestinal, and respiratory conditions.

In Verón, there are two clinics - the Verón Polytecnic Clinic and the Oscar de la Renta Pediatric Clinic. These two clinics are the main source of health services to the urban area. The Oscar de la Renta Pediatric Clinic opened in May 2017 and currently operates as a free clinic for children living in Verón. This children's clinic is expected to see around 30,000 children within the first year according to the local doctors. The clinic is funded by the Fundación Grupo Puntacana, the social responsibility entity for Grupo Puntacana, which operates Puntacana Resort and Club. The clinic's services include a pharmacy, a dentist and emergency center to provide comprehensive treatment of health. The clinic operates five days a week and employs doctors and nurses. As a new clinic, many of the services provided are tertiary level of prevention and focus on treatment rather than prevention. The clinic mainly serves children with ages ranging from infant to approximately 12 years old.

\section{Description of Project}

The objective of the project was to create a tool for the children's clinic and community leaders to use to promote a primary level of prevention for health issues that impact children. Working in coordination with the children's clinic staff, the resulting videos were played in the waiting room, so parents could view the messages while they waited to be seen. Since the videos were to focus on children health concerns, the videos were to be targeted at parents, both mothers and fathers, who are primary caregivers of children in the context of Verón. In addition, cultural dimensions of the patient population needed to be explored in order to ensure the appropriateness of the messaging, since patients had both Haitian and Dominican heritage. 


\section{Methodology}

The first phase of the project involved collecting qualitative data in the form of narratives and observations regarding the health concerns, cultural practices, and social function of Verón in the children's clinic. We met with the clinic doctors to discuss what major health cases and concerns their patients present with. This dialogue allowed us to begin to brainstorm health topics that could be explored in the health prevention videos. From there, we met with community leaders and residents from the 24 sectors in Verón to discuss their lifestyles and their perspective of health in their community. These narratives helped frame the cultural and environmental context surrounding the main health concerns of the community. The final component in this phase was to observe the children's clinic during operating hours. This allowed us to understand how health education was disseminated and to see doctor-patient interaction. In addition, this observation component was important to understand the setting and environment these prevention videos could be played in.

The second phase was to create a storyboard for the health prevention videos. Within these storyboards, we identified the target audience, the focal health issues, and the preventive messaging component. We created four storyboards for the films to present to the children's clinic doctors, which covered vaccinations, injury prevention, skin infections, and nutrition. After gaining feedback from the doctors on preventive practices and behaviors, we edited the storyboards accordingly.

The third phase included filming in several communities. We went to four sectors of Verón Villa la Fe, Pueblo Nuevo, Villa Esperanza, and Las Flores (see figure 2) - and filmed children and parents in the community. While we aimed to mostly film daily life as it played out, we did have to set some of the scenes to convey the message we intended. In addition, we gained permission from the children's clinic to film some of the patients (and their parents) and their conditions within the clinic setting. 


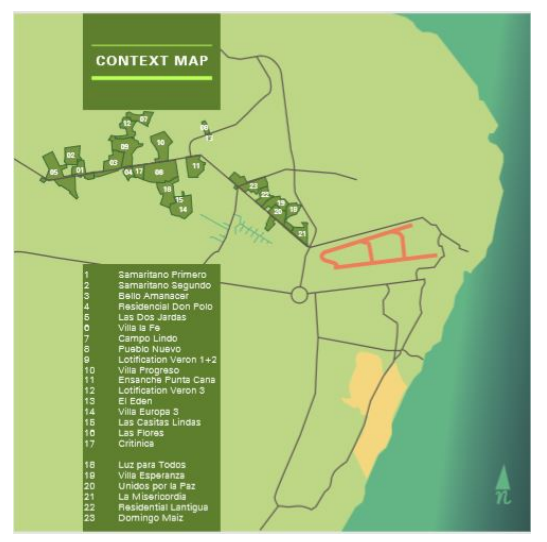

Figure 2: Perez, Carlos (2016), Map of Verón.

The fourth phase included compiling various footage from the different locations to edit and cut to create the three health prevention films. We had a fourth video outlined for nutrition. However, this video was eliminated from the collection due to lack of substantive footage. We aligned the videos to create a master cut and incorporated music and added Spanish subtitles to the film that were created by a fellow bilingual student.

The fifth phase was the evaluation of the project. When the videos were completed, we had the videos reviewed by the clinic doctors and a Vice President of Fundación Grupo Puntacana to ensure the video content, usage of text, and images were appropriate and aligned with the needs and culture of the community. We met with the clinic doctors to discuss the videos, and as we showcased each short video, the doctors would make suggestions on words to change and their thoughts on the images and flow of the content. The doctors reviewed any text in the video to ensure the language was appropriate to the community setting. By having the clinic doctors review the language component of the videos, we were able to ensure that the language used was consistent with how people communicate in the communities. Each video was reviewed by two clinic doctors to increase feedback on the videos before showing the videos to the community. The Vice President of Fundación Grupo Puntacana sent feedback via email regarding some of the imaging of the scenes from the injury prevention video. With his feedback, we decided to reedit some scenes with timing to improve the flow of the sequences.

The final phase was the dissemination of the videos. Once the revisions were complete and approved by the clinic staff and Fundación Grupo Puntacana, they were then played in the waiting room during the operating hours of the clinic. In addition, we had the opportunity to share the clinic videos with the Mayor of Verón. 


\section{Results}

The research project produced three health education videos, which focused on prevention across the three topical areas. The first video focused on prevention of skin infections. After having dialogue with the doctors and community members, the most common health concern brought to the free children's clinic was skin lesions and rashes. This video contained a series of scenarios in order to promote behaviors to prevent these skin lesions. Half the scenarios are negative behaviors that demonstrated potential causes to skin lesions while the other half were positive behaviors. The negative behavior scenarios included a boy playing in the dirt with no shoes or shirt and a boy walking in the street with no shoes in the dust and unpaved roads. The positive behavior scenarios included a mother sweeping the floor to remove dirt and dust from the house and a woman boiling water to wash hands.

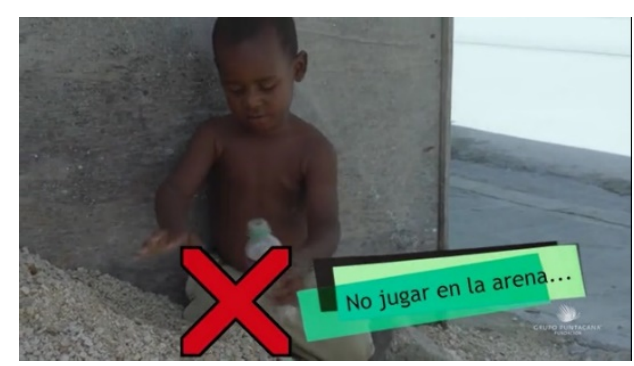

Figure 3: Evans, James (2017), Scene of boy playing in the dirt with no shoes or shirt.

The second video was a combination of health education and public service announcement in promotion of vaccinations. This short film included the purpose of vaccines and the importance of maintaining proper records for children by their parents. When having conversations with the doctors within the Oscar De La Renta pediatric clinic, the doctors requested a vaccination video because they found that many parents did not follow the standard steps for vaccinations. Oftentimes, the parents would vaccinate their child once, but do not come back for the follow-up vaccinations. In addition, some parents did not know the purpose of vaccinations. This video was a tool for the children's clinic to explain parents should get vaccinations for their children and why vaccinations are important. 


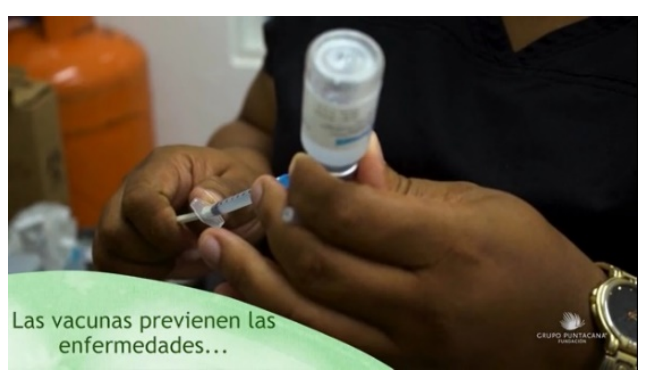

Figure 4: Evans, James (2017), Scene of clinic nurse preparing a vaccination.

The third film was about general safety tips to prevent unintentional injuries. The video contained a series of four scenarios from common unintentional injuries in Verón. The first scenario showed children looking both ways before crossing the street. This scene addressed the increased number of preventable injuries caused from pedestrian-motor vehicle injuries in the community. The second scenario included a mother intervening when a young boy tried to touch a hot stove. This scene addressed the increased cases of burns from kitchen accidents. The third scene showed a mother putting a baby into a crib. This scene addressed the health concerns from the doctors of infant fatalities from improper use of cribs. The final scenario was a group of young boys standing by a bucket of old, standing water. This scene addressed a cause of many of the increased cases of skin infections presented to doctors of the clinic.

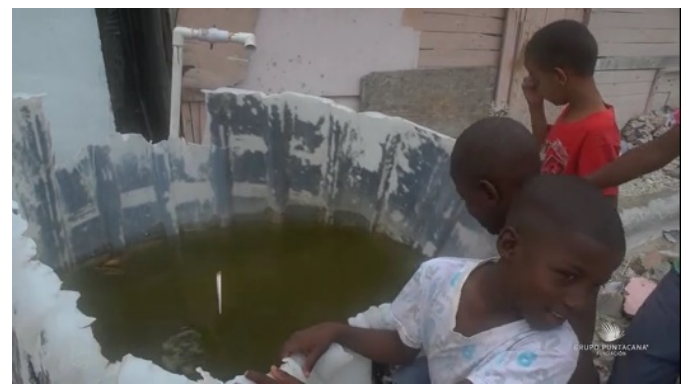

Figure 5: Evans, James (2017), Scene of children playing next to standing water.

Since our time in Verón was limited to filming the videos, we were unable to monitor and evaluate the impact of the videos on health outcomes in the community. However, with conversations with clinic staff regarding the videos after the final videos were presented, the clinic believed the videos would facilitate educating parents on important health issues that impact their children. The clinic was overcrowded as there was a low ratio of doctors to the 
number of children that come to the clinic on a daily occurrence. With limited time with each family, doctors wanted to have a different avenue to disseminate information to parents to shift from administering treatment to preventing major cases of various illness and disease. The doctors believed the medium of video could best meet this need.

\section{Discussion}

\section{Main Takeaway}

While the videos focused on the public health prevention dimension, education for the purposes of behavior change alone can only have a certain level of impact. Examining the social ecological model, the video can directly impact the individual level and, to an extent, the relationship level (Centers for Disease Control and Prevention, 2015). However, community and societal factors, such as governance, infrastructure, and environment, also play a key role in health (Centers for Disease Control and Prevention, 2015). For example, the condition of roads surfaces (paved versus unpaved) can be a contributing factor in injuries associated with traffic accidents, as well as exposure to poor air quality. Moreover, system-level infrastructure, such as that which provides access to water and safe disposal of waste are vital to decreasing certain burden of diseases. Without addressing these core, systems-level issues, health outcomes will continue to be poor in Verón.

\section{Limitations}

There were several limitations we faced when trying to create prevention-focused health education videos. The first limitation was language. As someone who could only speak beginner's Spanish, it was sometimes difficult to communicate. Utilizing the clinicians and a fellow bilingual student, I was dependent on their translations to communicate. In addition, being limited in language was a barrier in connecting with some of the community members. When trying to learn about daily life in Verón, not being able to directly communicate with community residents impacted community engagement with the film process.

Another barrier that we faced was the available technology in the children's clinic. Our initial ideation phase included several creative applications in the messaging. However, the clinic setup necessitated that the videos be shown without sound and on a screen that was smaller than anticipated. The stylistic choices we made had to account for limited sound and visual dimensions of the videos' projections. 
Moreover, during our initial meeting with the doctors, we were informed that many of the parents were challenged in their literacy and numeracy skills. To overcome these more fundamental barriers in health literacy, doctors tried to explain prevention techniques to parents one-on-one when they came in. However, with the high volume of patients, this was not an efficient use of time to ensure parents understood the proper follow-up steps or behaviors to prevent the same health outcomes from occurring in the future. Because of challenges with literacy among the parents of patients, the videos could not depend on written information.

Next, the prevention videos were created for the waiting room for the children's clinic. This is a limitation as the targeted population is already engaged with primary care. With prevention as the focus of the videos, the impact of the videos may be limited as the children and parents are at the clinic because they already have the injury. The videos may be better utilized in the community through community leaders to maximize the impact of the videos.

The filming process created some difficulties. We attempted to film rhythms and events already occurring in the communities to eliminate any staged nature of scenes. However, we did not observe every scene we had hoped to film, so we had to stage some of the scenes. While some communities were more open and willing to volunteer to participate in the film, we did encounter some reluctance in some of the communities we explored thus delaying our filming projects. Finally, staging scenes impacted how realistic the videos appeared to viewers. We had to film each staged scene several times to ensure it demonstrated an everyday occurrence.

Lastly, once the short films were produced and given to the clinic, we have no control of how the clinic utilized the videos. We cannot monitor the clinic staff to ensure they were playing the video in the children's clinic waiting room during hours of operation. This could limit the utilization and potential impact of the videos created.

\section{Next Steps}

The municipality of Verón is working to address many of the central issues that lead to the major health impacts among the children in Verón. With plans to pave roads and create a municipal water system, many of the health concerns that present at the children's clinic could decrease with these changes to system-level infrastructure. Yet, the public health prevention videos such as those that we created could positively impact health outcomes among their target populations if there were a strategic dissemination component that could be used to increase the reach of the video. Through community leaders, the videos could be disseminated through various channels 
such as WhatsApp or other social media platforms. This allows the information from the film to be available to more people in Verón rather than limited to the population that comes to the children's clinic because of a known health condition. For public health messaging to have the best impact, the message must engage the community (Krisberg, 2014). By disseminating the videos to community leaders, the message is not only available for more individuals, but the video can be a tool to engage the community in dialogue to make steps toward improving their health outcomes.

\section{Acknowledgments}

I would like to acknowledge Fundación Grupo Puntacana for their assistance and accommodating the team during our stay in the Dominican Republic. I would like to acknowledge James E. Evans, Jr for his assistance in filming and directing the videos. I would like to acknowledge Dr. Jocelyn Widmer for her guidance and support for this project.

\section{References}

Centers for Disease Control and Prevention. (2015). The social-ecological model: A framework for prevention. Atlanta, GA: CDC. Retrieved from https://www. $c d c$. gov/violenceprevention/overview/social-ecologicalmodel. $\mathrm{html}$.

De Leon, E., Fuentes, L. W., \& Cohen, J. E. (2014). Characterizing Periodic Messaging Interventions Across Health Behaviors and Media: Systematic Review. Journal of Medical Internet Research, 16(3), e93. http://doi.org/10.2196/jmir.2837

Golden, S. D., \& Earp, J. A. L. (2012). Social ecological approaches to individuals and their contexts: twenty years of health education \& behavior health promotion interventions. Health Education \& Behavior, 39(3), 364-372

Human Development Reports. (2016). Retrieved April 13, 2018, from http://www.hdr.undp.org/en/countries/profiles/DOM

Krisberg, K. (2014). Public health messaging: How it is said can influence behaviors: Beyond the facts. The Nation's Health., 44 (6) 1-20

Lewis, K. D. (2017). Utilizing perspectives from HIV-infected women, male partners and healthcare providers to design family planning mobile health messaging in Kenya: a qualitative study (Doctoral dissertation).

Wong, R., Harris, J. K., Staub, M., \& Bernhardt, J. M. (2017). Local health departments tweeting about Ebola: characteristics and messaging. Journal of Public Health Management and Practice, 23(2), e16-e24. 